\title{
Underlying Simple Graphs
}

\author{
Sebastian Koch (D) \\ Johannes Gutenberg University \\ Mainz, Germany $\left.\right|^{1}$
}

Summary. In this article the notion of the underlying simple graph of a graph (as defined in [8]) is formalized in the Mizar system [5], along with some convenient variants. The property of a graph to be without decorators (as introduced in [7]) is formalized as well to serve as the base of graph enumerations in the future.

MSC: 68T99 03B35 05C76

Keywords: graph operations; underlying simple graph

MML identifier: GLIB_009, version: 8.1.09 5.59.1363

\section{INTRODUCTION}

In the Mizar Mathematical Library [2] there are several formalizations of graphs with a varying degree of generality, see [1, [6], [10, [8, [9]. The GLIB series (starting with [8]) formalizes general digraphs (that is, digraphs with loops and parallel edges allowed) in Mizar [5] and provides a rich notation so that any digraph in Mizar can be seen as an undirected graph simply by ignoring the direction of the edges (although they are always there). In conclusion, there is no need for another formalization of undirected graphs, in contrast to how it is typically done in the literature (cf. [12, 3]), and the underlying (undirected) graph of a digraph (in the sense of [8]) is itself. For undirected graphs or digraphs possibly containing loops and multiple parallel edges, the underlying (simple) graph or digraph is derived by removing the loops and replacing each

\footnotetext{
${ }^{1}$ The author is enrolled in the Johannes Gutenberg University in Mayence, Germany, mailto: skoch02@students. uni-mainz.de 
set of parallel edges with a single edge. That concept requires formalization and this article provides subgraph modes that respectively remove loops, (directed) parallel edges or both from a given (di)graph. "Much of graph theory is concerned with the study of simple graphs" [4, p. 3] which results in many books only studying simple graphs, even when graphs are more generally introduced in the respective book (for example [11]).

The rather extensive preliminaries contain many theorems that would fit well into earlier articles of the GLIB series, for example:

- The source and target of a directed edge in a graph are uniquely determined.

- A walk in a graph is uniquely determined by its vertex and edge sequence.

- Adding vertices to a graph doesn't change adjacencies.

The next section introduces plain graphs. Graphs, as defined in [8], can arbitrarily be expanded with decorators as done in [7]. Therefore for any non empty set $S$ the set containing all graphs with vertex and edge sets contained in $S$ does not exist because of possible decorators, even if $S$ only contains a single element. A graph is called plain if it does not contain additional decorators, and then the set of all plain graphs with vertex and edge sets contained in $S$ can be constructed, which will be needed for graph enumeration at a later point in time.

In the section after that the set of all loops of a graph is introduced as well as a graph operator removing all loops from a given graph as a special case of removing edges.

At the start of the following section, two equivalence relations are defined on the edge set, where two edges are equivalent iff they are (directed) parallel. Then modes are introduced to pick one edge out of each set of (directed) parallel edges. Using such representative edge selections, the graphs with parallel edges removed can be defined as induced subgraphs. While the directed and undirected variants are formalized along each other, there are also some theorems focusing on how they interact with each other.

This trend is continued in the last section, where the underlying simple graphs are introduced as induced subgraphs on the representative edge selections with the loops removed. Naturally, these subgraphs can also be constructed by removing loops and then parallel edges from a graph or vice versa. 


\section{Preliminaries}

Now we state the propositions:

(1) Let us consider sets $X, Y$. If $Y \subseteq X$, then $X \backslash(X \backslash Y)=Y$.

(2) Let us consider a binary relation $R$, and a set $X$. Then

(i) $\left(R\lceil X)^{\smile}=X 1 R^{\smile}\right.$, and

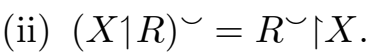

Let us consider a function $f$ and a set $Y$. Now we state the propositions:

(3) $\operatorname{dom}(Y 1 f)=f^{-1}(Y)$.

Proof: For every object $x, x \in \operatorname{dom}(Y 1 f)$ iff $x \in f^{-1}(Y)$.

(4) $Y 1 f=f\lceil\operatorname{dom}(Y 1 f)$. The theorem is a consequence of (3).

(5) Let us consider a one-to-one function $f$, and a set $X$. Then

(i) $\left(f\lceil X)^{-1}=X 1 f^{-1}\right.$, and

(ii) $(X \mid f)^{-1}=f^{-1} \uparrow X$.

The theorem is a consequence of (2).

(6) Let us consider a graph $G$, and objects $e, x_{1}, y_{1}, x_{2}, y_{2}$. Suppose $e$ joins $x_{1}$ to $y_{1}$ in $G$ and $e$ joins $x_{2}$ to $y_{2}$ in $G$. Then

(i) $x_{1}=x_{2}$, and

(ii) $y_{1}=y_{2}$.

Let $G$ be a trivial graph. Let us observe that the vertices of $G$ is trivial and every graph which is trivial and non-directed-multi is also non-multi.

Let $G$ be a trivial, non-directed-multi graph. Let us observe that the edges of $G$ is trivial.

Now we state the propositions:

(7) Let us consider a graph $G$, sets $X, Y$, and objects $e, x, y$. Suppose $e$ joins $x$ to $y$ in $G$ and $x \in X$ and $y \in Y$. Then $e$ joins a vertex from $X$ to a vertex from $Y$ in $G$.

(8) Let us consider a trivial graph $G$, and a graph $H$. Suppose the vertices of $H \subseteq$ the vertices of $G$ and the edges of $H \subseteq$ the edges of $G$. Then $H$ is trivial and subgraph of $G$.

(9) Let us consider a graph $G$. Then $G \approx G \uparrow$ (the graph selectors).

Let us consider a graph $G$, sets $X, Y$, and an object $e$. Now we state the propositions:

(10) $e$ joins a vertex from $X$ and a vertex from $Y$ in $G$ if and only if $e$ joins a vertex from $Y$ and a vertex from $X$ in $G$. 
(11) $e$ joins a vertex from $X$ and a vertex from $Y$ in $G$ if and only if $e$ joins a vertex from $X$ to a vertex from $Y$ in $G$ or $e$ joins a vertex from $Y$ to a vertex from $X$ in $G$.

Let us consider a graph $G$ and objects $e, v, w$. Now we state the propositions:

(12) If $e$ joins a vertex from $\{v\}$ and a vertex from $\{w\}$ in $G$, then $e$ joins $v$ and $w$ in $G$.

(13) If $e$ joins a vertex from $\{v\}$ to a vertex from $\{w\}$ in $G$, then $e$ joins $v$ to $w$ in $G$.

(14) Let us consider a graph $G$, and objects $v, w$. Suppose $v \neq w$. Then

(i) G.edgesDBetween $(\{v\},\{w\})$ misses $G$.edgesDBetween $(\{w\},\{v\})$, and

(ii) G.edgesBetween $(\{v\},\{w\})=G$.edgesDBetween $(\{v\},\{w\}) \cup$ G.edgesDBetween $(\{w\},\{v\})$.

The theorem is a consequence of (11).

(15) Let us consider a graph $G$, and a set $X$. Then $G$.edgesBetween $(X, X)=$ $G$.edgesDBetween $(X, X)$. The theorem is a consequence of (11).

(16) Let us consider a graph $G$, and sets $X, Y$. Then $G$.edgesBetween $(X, Y)=$ $G$.edgesBetween $(Y, X)$. The theorem is a consequence of (10).

Let us consider a graph $G$. Now we state the propositions:

(17) $G$ is loopless if and only if for every object $v$, there exists no object $e$ such that $e$ joins $v$ to $v$ in $G$.

Proof: For every object $v$, there exists no object $e$ such that $e$ joins $v$ and $v$ in $G$.

(18) $G$ is loopless if and only if for every object $v$, there exists no object $e$ such that $e$ joins a vertex from $\{v\}$ and a vertex from $\{v\}$ in $G$.

ProOF: For every object $v$, there exists no object $e$ such that $e$ joins $v$ and $v$ in $G$.

(19) $G$ is loopless if and only if for every object $v$, there exists no object $e$ such that $e$ joins a vertex from $\{v\}$ to a vertex from $\{v\}$ in $G$. The theorem is a consequence of (11) and (18).

(20) $G$ is loopless if and only if for every object $v, G$.edgesBetween $(\{v\},\{v\})=$ $\emptyset$. The theorem is a consequence of (18).

(21) $G$ is loopless if and only if for every object $v, G$.edgesDBetween $(\{v\},\{v\})=$ $\emptyset$. The theorem is a consequence of (19).

Let $G$ be a loopless graph and $v$ be an object. One can verify that $G$.edgesBetween $(\{v\},\{v\})$ is empty and $G$.edgesDBetween $(\{v\},\{v\})$ is empty. 
(22) Let us consider a graph $G$. Then $G$ is non-multi if and only if for every objects $v, w, G$.edgesBetween $(\{v\},\{w\})$ is trivial. The theorem is a consequence of $(12)$.

Let $G$ be a non-multi graph and $v, w$ be objects. One can verify that $G$.edgesBetween $(\{v\},\{w\})$ is trivial. Now we state the proposition:

(23) Let us consider a graph $G$. Then $G$ is non-directed-multi if and only if for every objects $v, w, G$.edgesDBetween $(\{v\},\{w\})$ is trivial. The theorem is a consequence of (13) and (7).

Let $G$ be a non-directed-multi graph and $v, w$ be objects. One can check that $G$.edgesDBetween $(\{v\},\{w\})$ is trivial.

Let $G$ be a non trivial graph. Let us note that every subgraph of $G$ which is spanning is also non trivial.

Let $G$ be a graph. One can check that every vertex of $G$ which is isolated is also non endvertex.

Let us consider a graph $G$ and a vertex $v$ of $G$. Now we state the propositions:

(24) (G.walkOf $(v))$.edgeSeq ()$=\varepsilon_{\alpha}$, where $\alpha$ is the edges of $G$.

(25) (G.walkOf $(v))$.edges ()$=\emptyset$. The theorem is a consequence of $(24)$.

Let $G$ be a graph and $W$ be a trivial walk of $G$. Note that $W \cdot \operatorname{edges}()$ is empty and trivial.

Let $W$ be a walk of $G$. Note that $W$.vertices () is non empty.

Now we state the propositions:

(26) Let us consider graphs $G_{1}, G_{2}$, a walk $W_{1}$ of $G_{1}$, and a walk $W_{2}$ of $G_{2}$. Suppose $W_{1}$.vertexSeq ()$=W_{2} \cdot \operatorname{vertexSeq}()$ and $W_{1}$.edgeSeq ()$=$ $W_{2}$.edgeSeq(). Then $W_{1}=W_{2}$.

Proof: For every natural number $n$ such that $1 \leqslant n \leqslant$ len $W_{1}$ holds $W_{1}(n)=W_{2}(n)$.

(27) Let us consider a graph $G$, a finite sequence $p$ of elements of the vertices of $G$, and a finite sequence $q$ of elements of the edges of $G$. Suppose len $p=1+\operatorname{len} q$ and for every element $n$ of $\mathbb{N}$ such that $1 \leqslant n$ and $n+1 \leqslant \operatorname{len} p$ holds $q(n)$ joins $p(n)$ and $p(n+1)$ in $G$. Then there exists a walk $W$ of $G$ such that

(i) $W \cdot \operatorname{vertexSeq}()=p$, and

(ii) $W \cdot \operatorname{edgeSeq}()=q$.

Proof: Define $\mathcal{P}$ [object, object] $\equiv$ there exists a natural number $m$ such that $m=\$_{1}$ and if $m$ is odd, then $\$_{2}=p(m+1 \operatorname{div} 2)$ and if $m$ is even, then $\$_{2}=q(m \operatorname{div} 2)$. For every natural number $k$ such that $k \in \operatorname{Seg}(\operatorname{len} p+\operatorname{len} q)$ there exists an element $x$ of (the vertices of $G) \cup($ the edges of $G$ ) such that $\mathcal{P}[k, x]$. Consider $W$ being a finite sequence of elements of (the vertices of 
$G) \cup($ the edges of $G)$ such that $\operatorname{dom} W=\operatorname{Seg}(\operatorname{len} p+\operatorname{len} q)$ and for every natural number $k$ such that $k \in \operatorname{Seg}(\operatorname{len} p+\operatorname{len} q)$ holds $\mathcal{P}[k, W(k)] . W(1) \in$ the vertices of $G$. For every odd element $n$ of $\mathbb{N}$ such that $n<$ len $W$ holds $W(n+1)$ joins $W(n)$ and $W(n+2)$ in $G$. For every natural number $k$ such that $1 \leqslant k \leqslant \operatorname{len} p$ holds $p(k)=(W$.vertexSeq ()$)(k)$. For every natural number $k$ such that $1 \leqslant k \leqslant$ len $q$ holds $q(k)=(W$.edgeSeq ()$)(k)$.

(28) Let us consider a graph $G$, and a walk $W$ of $G$. Then len $(W$.vertexSeq ()$)=$ $W$.length ()$+1$.

(29) Let us consider graphs $G_{1}, G_{2}$, a walk $W_{1}$ of $G_{1}$, a walk $W_{2}$ of $G_{2}$, and an odd natural number $n$. If $W_{1} \cdot \operatorname{vertexSeq}()=W_{2} \cdot \operatorname{vertexSeq}()$, then $W_{1}(n)=W_{2}(n)$.

Let us consider graphs $G_{1}, G_{2}$, a walk $W_{1}$ of $G_{1}$, and a walk $W_{2}$ of $G_{2}$. Now we state the propositions:

(30) Suppose $W_{1} \cdot \operatorname{vertexSeq}()=W_{2} \cdot \operatorname{vertexSeq}()$. Then

(i) len $W_{1}=$ len $W_{2}$, and

(ii) $W_{1} \cdot \operatorname{length}()=W_{2} \cdot \operatorname{length}()$, and

(iii) $W_{1}$.first ()$=W_{2}$.first $($ ), and

(iv) $W_{1}$.last ()$=W_{2}$.last () , and

(v) $W_{2}$ is walk from $W_{1}$.first() to $W_{1}$.last().

The theorem is a consequence of (29).

(31) If $W_{1} \cdot \operatorname{vertexSeq}()=W_{2}$.vertexSeq () , then if $W_{1}$ is not trivial, then $W_{2}$ is not trivial and if $W_{1}$ is closed, then $W_{2}$ is closed. The theorem is a consequence of $(30)$.

(32) Suppose $W_{1} \cdot \operatorname{vertexSeq}()=W_{2} \cdot \operatorname{vertexSeq}()$ and len $W_{1} \neq 5$. Then

(i) if $W_{1}$ is path-like, then $W_{2}$ is path-like, and

(ii) if $W_{1}$ is cycle-like, then $W_{2}$ is cycle-like.

Proof: If $W_{1}$ is path-like, then $W_{2}$ is path-like.

The scheme IndWalk deals with a graph $\mathcal{G}$ and a unary predicate $\mathcal{P}$ and states that

(Sch. 1) For every walk $W$ of $\mathcal{G}, \mathcal{P}[W]$

provided

- for every trivial walk $W$ of $\mathcal{G}, \mathcal{P}[W]$ and

- for every walk $W$ of $\mathcal{G}$ and for every object $e$ such that $e \in W$.last().edgesInOut() and $\mathcal{P}[W]$ holds $\mathcal{P}[W$.addEdge $(e)]$. 
The scheme IndDWalk deals with a graph $\mathcal{G}$ and a unary predicate $\mathcal{P}$ and states that

(Sch. 2) For every dwalk $W$ of $\mathcal{G}, \mathcal{P}[W]$

provided

- for every trivial dwalk $W$ of $\mathcal{G}, \mathcal{P}[W]$ and

- for every dwalk $W$ of $\mathcal{G}$ and for every object $e$ such that

$e \in W$.last().edgesOut() and $\mathcal{P}[W]$ holds $\mathcal{P}[W$.addEdge $(e)]$.

Now we state the propositions:

(33) Let us consider a graph $G_{1}$, a subset $E$ of the edges of $G_{1}$, and a subgraph $G_{2}$ of $G_{1}$ induced by the vertices of $G_{1}$ and $E$. If $G_{2}$ is connected, then $G_{1}$ is connected.

(34) Let us consider a graph $G_{1}$, a set $E$, and a subgraph $G_{2}$ of $G_{1}$ with edges $E$ removed. If $G_{2}$ is connected, then $G_{1}$ is connected.

Let $G_{1}$ be a non connected graph and $E$ be a set. One can check that every subgraph of $G_{1}$ with edges $E$ removed is non connected.

(35) Let us consider a graph $G_{1}$, and a subgraph $G_{2}$ of $G_{1}$. Suppose for every walk $W_{1}$ of $G_{1}$, there exists a walk $W_{2}$ of $G_{2}$ such that $W_{2}$ is walk from $W_{1}$.first() to $W_{1}$.last(). Let us consider a vertex $v_{1}$ of $G_{1}$, and a vertex $v_{2}$ of $G_{2}$. If $v_{1}=v_{2}$, then $G_{1}$.reachableFrom $\left(v_{1}\right)=G_{2}$.reachableFrom $\left(v_{2}\right)$.

(36) Let us consider a graph $G_{1}$, and a subgraph $G_{2}$ of $G_{1}$. Suppose for every walk $W_{1}$ of $G_{1}$, there exists a walk $W_{2}$ of $G_{2}$ such that $W_{2}$ is walk from $W_{1}$.first() to $W_{1}$.last(). If $G_{1}$ is connected, then $G_{2}$ is connected.

Let us consider a graph $G_{1}$ and a spanning subgraph $G_{2}$ of $G_{1}$. Now we state the propositions:

(37) Suppose for every vertex $v_{1}$ of $G_{1}$ and for every vertex $v_{2}$ of $G_{2}$ such that $v_{1}=v_{2}$ holds $G_{1}$.reachableFrom $\left(v_{1}\right)=G_{2} \cdot \operatorname{reachableFrom}\left(v_{2}\right)$. Then $G_{1} \cdot \operatorname{componentSet}()=G_{2} \cdot \operatorname{componentSet}()$.

(38) Suppose for every vertex $v_{1}$ of $G_{1}$ and for every vertex $v_{2}$ of $G_{2}$ such that $v_{1}=v_{2}$ holds $G_{1}$.reachableFrom $\left(v_{1}\right)=G_{2}$.reachableFrom $\left(v_{2}\right)$. Then $G_{1}$.numComponents ()$=G_{2}$.numComponents () . The theorem is a consequence of (37).

(39) Let us consider a graph $G$. Then $G$ is loopless if and only if for every vertex $v$ of $G, v$ and $v$ are not adjacent.

Let $G$ be a non complete graph. One can check that every subgraph of $G$ which is spanning is also non complete.

Now we state the propositions: 
(40) Let us consider graphs $G_{2}, G_{3}$, and a supergraph $G_{1}$ of $G_{3}$. If $G_{1} \approx G_{2}$, then $G_{2}$ is a supergraph of $G_{3}$.

(41) Let us consider a graph $G_{2}$, a set $V$, a supergraph $G_{1}$ of $G_{2}$ extended by the vertices from $V$, sets $x, y$, and an object $e$. Then

(i) $e$ joins $x$ and $y$ in $G_{1}$ iff $e$ joins $x$ and $y$ in $G_{2}$, and

(ii) $e$ joins $x$ to $y$ in $G_{1}$ iff $e$ joins $x$ to $y$ in $G_{2}$, and

(iii) $e$ joins a vertex from $x$ and a vertex from $y$ in $G_{1}$ iff $e$ joins a vertex from $x$ and a vertex from $y$ in $G_{2}$, and

(iv) $e$ joins a vertex from $x$ to a vertex from $y$ in $G_{1}$ iff $e$ joins a vertex from $x$ to a vertex from $y$ in $G_{2}$.

(42) Let us consider graphs $G_{1}, G_{2}$. Suppose $G_{1} \approx G_{2}$. Then $G_{2}$ is a graph given by reversing directions of the edges $\emptyset$ of $G_{1}$.

(43) Every graph is a graph given by reversing directions of the edges $\emptyset$ of $G$.

\section{Plain Graphs}

Let $G$ be a graph. We say that $G$ is plain if and only if

(Def. 1) $\operatorname{dom} G=$ the graph selectors.

Note that $G \uparrow$ (the graph selectors) is plain.

Let $V$ be a non empty set, $E$ be a set, and $S, T$ be functions from $E$ into $V$. Let us observe that createGraph $(V, E, S, T)$ is plain.

Let $G$ be a graph and $X$ be a set. Note that $G$.set(WeightSelector, $X$ ) is non plain and $G$.set(ELabelSelector, $X$ ) is non plain and $G$.set(VLabelSelector, $X$ ) is non plain and there exists a graph which is plain.

Now we state the proposition:

(44) Let us consider plain graphs $G_{1}, G_{2}$. If $G_{1} \approx G_{2}$, then $G_{1}=G_{2}$.

Let $G$ be a graph. Note that there exists a subgraph of $G$ which is plain.

Let $V$ be a set. One can check that there exists a subgraph of $G$ with vertices $V$ removed which is plain.

Let $E$ be a set. Let us note that there exists a subgraph of $G$ induced by $V$ and $E$ which is plain and there exists a subgraph of $G$ with edges $E$ removed which is plain and there exists a graph given by reversing directions of the edges $E$ of $G$ which is plain.

Let $v$ be a set. One can verify that there exists a subgraph of $G$ with vertex $v$ removed which is plain.

Let $e$ be a set. One can verify that there exists a subgraph of $G$ with edge $e$ removed which is plain and there exists a supergraph of $G$ which is plain. 
Let $V$ be a set. Let us note that there exists a supergraph of $G$ extended by the vertices from $V$ which is plain.

Let $v, e, w$ be objects. One can check that there exists a supergraph of $G$ extended by $e$ between vertices $v$ and $w$ which is plain and there exists a supergraph of $G$ extended by $v, w$ and $e$ between them which is plain.

Let $v$ be an object and $V$ be a set. Let us note that there exists a supergraph of $G$ extended by vertex $v$ and edges from $V$ of $G$ to $v$ which is plain and there exists a supergraph of $G$ extended by vertex $v$ and edges from $v$ to $V$ of $G$ which is plain and there exists a supergraph of $G$ extended by vertex $v$ and edges between $v$ and $V$ of $G$ which is plain.

\section{Graphs with Loops Removed}

Let $G$ be a graph. The functor $G$.loops() yielding a subset of the edges of $G$ is defined by

(Def. 2) for every object $e, e \in i$ iff there exists an object $v$ such that $e$ joins $v$ and $v$ in $G$.

Now we state the propositions:

(45) Let us consider a graph $G$, and an object $e$. Then $e \in G$.loops() if and only if there exists an object $v$ such that $e$ joins $v$ to $v$ in $G$.

(46) Let us consider a graph $G$, and objects $e, v, w$. If $e$ joins $v$ and $w$ in $G$ and $v \neq w$, then $e \notin G$.loops () .

(47) Let us consider a graph $G$. Then $G$ is loopless if and only if $G \cdot \operatorname{loops}()=\emptyset$.

Let $G$ be a loopless graph. Let us observe that $G$.loops() is empty.

Let $G$ be a non loopless graph. Let us observe that $G \cdot \operatorname{loops}()$ is non empty.

Now we state the propositions:

(48) Let us consider a graph $G_{1}$, and a subgraph $G_{2}$ of $G_{1}$. Then $G_{2} \cdot \operatorname{loops}() \subseteq$ $G_{1} \cdot \operatorname{loops}()$. The theorem is a consequence of (45).

(49) Let us consider a graph $G_{2}$, and a supergraph $G_{1}$ of $G_{2}$. Then $G_{2} \cdot \operatorname{loops}() \subseteq$ $G_{1} \cdot \operatorname{loops}()$. The theorem is a consequence of (48).

(50) Let us consider graphs $G_{1}, G_{2}$. If $G_{1} \approx G_{2}$, then $G_{1} \cdot \operatorname{loops}()=G_{2} \cdot \operatorname{loops}()$. The theorem is a consequence of (48).

(51) Let us consider a graph $G_{1}$, a set $E$, and a graph $G_{2}$ given by reversing directions of the edges $E$ of $G_{1}$. Then $G_{1} \cdot \operatorname{loops}()=G_{2} \cdot \operatorname{loops}()$.

(52) Let us consider a graph $G_{2}$, a set $V$, and a supergraph $G_{1}$ of $G_{2}$ extended by the vertices from $V$. Then $G_{1} \cdot \operatorname{loops}()=G_{2} \cdot \operatorname{loops}()$. The theorem is a consequence of (41) and (49). 
(53) Let us consider a graph $G_{2}$, objects $v_{1}, e, v_{2}$, and a supergraph $G_{1}$ of $G_{2}$ extended by $e$ between vertices $v_{1}$ and $v_{2}$. If $v_{1} \neq v_{2}$, then $G_{1} \cdot \operatorname{loops}()=$ $G_{2} \cdot \operatorname{loops}()$. The theorem is a consequence of (50) and (49).

(54) Let us consider a graph $G_{2}$, a vertex $v$ of $G_{2}$, an object $e$, and a supergraph $G_{1}$ of $G_{2}$ extended by $e$ between vertices $v$ and $v$. Suppose $e \notin$ the edges of $G_{2}$. Then $G_{1} \cdot \operatorname{loops}()=G_{2} \cdot \operatorname{loops}() \cup\{e\}$. The theorem is a consequence of (45) and (49).

(55) Let us consider a graph $G_{2}$, objects $v_{1}, e, v_{2}$, and a supergraph $G_{1}$ of $G_{2}$ extended by $v_{1}, v_{2}$ and $e$ between them. Then $G_{1} \cdot \operatorname{loops}()=G_{2} \cdot \operatorname{loops}()$. The theorem is a consequence of (49) and (50).

(56) Let us consider a graph $G_{2}$, an object $v$, a set $V$, and a supergraph $G_{1}$ of $G_{2}$ extended by vertex $v$ and edges between $v$ and $V$ of $G_{2}$. Then $G_{1} \cdot \operatorname{loops}()=G_{2} \cdot \operatorname{loops}()$. The theorem is a consequence of (49) and (50).

(57) Let us consider a graph $G$, and a path $P$ of $G$. Then

(i) P.edges() misses G.loops(), or

(ii) there exist objects $v, e$ such that $e$ joins $v$ and $v$ in $G$ and $P=$ $G$.walkOf $(v, e, v)$.

Let $G$ be a graph. A subgraph of $G$ with loops removed is a subgraph of $G$ with edges $G$.loops() removed. Now we state the proposition:

(58) Let us consider a loopless graph $G_{1}$, and a graph $G_{2}$. Then $G_{1} \approx G_{2}$ if and only if $G_{2}$ is a subgraph of $G_{1}$ with loops removed.

Let us consider graphs $G_{1}, G_{2}$ and a subgraph $G_{3}$ of $G_{1}$ with loops removed.

(59) $G_{2} \approx G_{3}$ if and only if $G_{2}$ is a subgraph of $G_{1}$ with loops removed.

(60) If $G_{1} \approx G_{2}$, then $G_{3}$ is a subgraph of $G_{2}$ with loops removed. The theorem is a consequence of (50).

Let $G$ be a graph. Observe that every subgraph of $G$ with loops removed is loopless and there exists a subgraph of $G$ with loops removed which is plain.

Let $G$ be a non-multi graph. Observe that every subgraph of $G$ with loops removed is simple.

Let $G$ be a non-directed-multi graph. One can check that every subgraph of $G$ with loops removed is directed-simple.

Let $G$ be a complete graph. Observe that every subgraph of $G$ with loops removed is complete.

Now we state the propositions:

(61) Let us consider a graph $G_{1}$, a subgraph $G_{2}$ of $G_{1}$ with loops removed, and a walk $W_{1}$ of $G_{1}$. Then there exists a walk $W_{2}$ of $G_{2}$ such that $W_{2}$ is walk from $W_{1}$.first() to $W_{1}$.last(). The theorem is a consequence of (57). 
(62) Let us consider a graph $G_{1}$, a subgraph $G_{2}$ of $G_{1}$ with loops removed, a vertex $v_{1}$ of $G_{1}$, and a vertex $v_{2}$ of $G_{2}$. If $v_{1}=v_{2}$, then $G_{1}$.reachableFrom $\left(v_{1}\right)=G_{2}$.reachableFrom $\left(v_{2}\right)$. The theorem is a consequence of (61) and (35).

Let $G$ be a connected graph. Observe that every subgraph of $G$ with loops removed is connected. Let $G$ be a non connected graph. Observe that every subgraph of $G$ with loops removed is non connected. Let us consider a graph $G_{1}$ and a subgraph $G_{2}$ of $G_{1}$ with loops removed. Now we state the propositions:

(63) $G_{1}$.componentSet ()$=G_{2}$.componentSet(). The theorem is a consequence of (62) and (37).

(64) $G_{1}$.numComponents ()$=G_{2}$.numComponents(). The theorem is a consequence of (62) and (38).

(65) $G_{1}$ is chordal if and only if $G_{2}$ is chordal. The theorem is a consequence of (46) and (57).

Let $G$ be a chordal graph. Let us observe that every subgraph of $G$ with loops removed is chordal. Now we state the proposition:

(66) Let us consider a graph $G_{1}$, a set $v$, a subgraph $G_{2}$ of $G_{1}$ with loops removed, and a subgraph $G_{3}$ of $G_{1}$ with vertex $v$ removed. Then every subgraph of $G_{2}$ with vertex $v$ removed is a subgraph of $G_{3}$ with loops removed. The theorem is a consequence of (1), (48), (59), and (60).

Let us consider a graph $G_{1}$, a subgraph $G_{2}$ of $G_{1}$ with loops removed, a vertex $v_{1}$ of $G_{1}$, and a vertex $v_{2}$ of $G_{2}$. Now we state the propositions:

(67) If $v_{1}=v_{2}$, then $v_{1}$ is cut-vertex iff $v_{2}$ is cut-vertex. The theorem is a consequence of (66) and (64).

(68) If $v_{1}=v_{2}$ and $v_{1}$ is endvertex, then $v_{2}$ is endvertex. The theorem is a consequence of (46).

\section{Graphs with Parallel Edges Removed}

Let $G$ be a graph. The functors: $\operatorname{EdgeParEqRel}(G)$ and $\operatorname{DEdgeParEqRel}(G)$ yielding equivalence relations of the edges of $G$ are defined by conditions

(Def. 3) for all objects $e_{1}, e_{2},\left\langle e_{1}, e_{2}\right\rangle \in \operatorname{EdgeParEqRel}(G)$ iff there exist objects $v_{1}, v_{2}$ such that $e_{1}$ joins $v_{1}$ and $v_{2}$ in $G$ and $e_{2}$ joins $v_{1}$ and $v_{2}$ in $G$,

(Def. 4) for all objects $e_{1}, e_{2},\left\langle e_{1}, e_{2}\right\rangle \in \operatorname{DEdgeParEqRel}(G)$ iff there exist objects $v_{1}, v_{2}$ such that $e_{1}$ joins $v_{1}$ to $v_{2}$ in $G$ and $e_{2}$ joins $v_{1}$ to $v_{2}$ in $G$, respectively.

Let us consider a graph $G$. Now we state the propositions:

(69) $\operatorname{DEdgeParEqRel}(G) \subseteq \operatorname{EdgeParEqRel}(G)$. 
(70) $G$ is non-multi if and only if $\operatorname{EdgeParEqRel}(G)=\mathrm{id}_{\alpha}$, where $\alpha$ is the edges of $G$.

(71) $G$ is non-directed-multi if and only if $\operatorname{DEdgeParEqRel}(G)=\operatorname{id}_{\alpha}$, where $\alpha$ is the edges of $G$.

Let $G$ be an edgeless graph. One can verify that $\operatorname{EdgeParEqRel}(G)$ is empty and DEdgeParEqRel $(G)$ is empty.

Let $G$ be a non edgeless graph. Observe that EdgeParEqRel $(G)$ is non empty and DEdgeParEqRel $(G)$ is non empty.

Let $G$ be a graph.

A representative selection of the parallel edges of $G$ is a subset of the edges of $G$ defined by

(Def. 5) for every objects $v, w, e_{0}$ such that $e_{0}$ joins $v$ and $w$ in $G$ there exists an object $e$ such that $e$ joins $v$ and $w$ in $G$ and $e \in i t$ and for every object $e^{\prime}$ such that $e^{\prime}$ joins $v$ and $w$ in $G$ and $e^{\prime} \in i t$ holds $e^{\prime}=e$.

A representative selection of the directed-parallel edges of $G$ is a subset of the edges of $G$ defined by

(Def. 6) for every objects $v, w, e_{0}$ such that $e_{0}$ joins $v$ to $w$ in $G$ there exists an object $e$ such that $e$ joins $v$ to $w$ in $G$ and $e \in i t$ and for every object $e^{\prime}$ such that $e^{\prime}$ joins $v$ to $w$ in $G$ and $e^{\prime} \in i t$ holds $e^{\prime}=e$.

Let $G$ be an edgeless graph. Let us observe that every representative selection of the parallel edges of $G$ is empty and every representative selection of the directed-parallel edges of $G$ is empty.

Let $G$ be a non edgeless graph. Let us observe that every representative selection of the parallel edges of $G$ is non empty and every representative selection of the directed-parallel edges of $G$ is non empty.

Now we state the propositions:

(72) Let us consider a graph $G$, and a representative selection of the directedparallel edges $E_{1}$ of $G$. Then there exists a representative selection of the parallel edges $E_{2}$ of $G$ such that $E_{2} \subseteq E_{1}$.

Proof: Set $A=\{\{e$, where $e$ is an element of the edges of $G: e$ joins $v_{1}$ and $v_{2}$ in $G$ and $\left.e \in E_{1}\right\}$, where $v_{1}, v_{2}$ are vertices of $G$ : there exists an object $e_{0}$ such that $e_{0}$ joins $v_{1}$ and $v_{2}$ in $\left.G\right\}$. Define $\mathcal{P}$ [object, object] $\equiv$ there exists a non empty set $S$ such that $\$_{1}=S$ and $\$_{2}=$ the element of $S$. For every object $x$ such that $x \in A$ there exists an object $y$ such that $\mathcal{P}[x, y]$. Consider $f$ being a function such that $\operatorname{dom} f=A$ and for every object $x$ such that $x \in A$ holds $\mathcal{P}[x, f(x)]$. For every object $e$ such that $e \in \operatorname{rng} f$ holds $e \in E_{1}$. Reconsider $E_{2}=\operatorname{rng} f$ as a subset of the edges of $G$. For every objects $v, w, e_{0}$ such that $e_{0}$ joins $v$ and $w$ in $G$ there exists an object $e$ such that $e$ joins $v$ and $w$ in $G$ and $e \in E_{2}$ and for every object 
$e^{\prime}$ such that $e^{\prime}$ joins $v$ and $w$ in $G$ and $e^{\prime} \in E_{2}$ holds $e^{\prime}=e$.

(73) Let us consider a graph $G$, and a representative selection of the parallel edges $E_{2}$ of $G$. Then there exists a representative selection of the directedparallel edges $E_{1}$ of $G$ such that $E_{2} \subseteq E_{1}$.

Proof: Set $A=\left\{\left\{e\right.\right.$, where $e$ is an element of the edges of $G: e$ joins $v_{1}$ to $v_{2}$ in $G$, where $v_{1}, v_{2}$ are vertices of $G$ : there exists an object $e_{0}$ such that $e_{0}$ joins $v_{1}$ to $v_{2}$ in $G$ and for every object $e_{0}$ such that $e_{0}$ joins $v_{1}$ to $v_{2}$ in $G$ holds $\left.e_{0} \notin E_{2}\right\}$. Define $\mathcal{P}$ [object, object] $\equiv$ there exists a non empty set $S$ such that $\$_{1}=S$ and $\$_{2}=$ the element of $S$. For every object $x$ such that $x \in A$ there exists an object $y$ such that $\mathcal{P}[x, y]$. Consider $f$ being a function such that $\operatorname{dom} f=A$ and for every object $x$ such that $x \in A$ holds $\mathcal{P}[x, f(x)]$. For every object $e$ such that $e \in \operatorname{rng} f$ holds $e \in$ the edges of $G$. Reconsider $E_{1}=E_{2} \cup \operatorname{rng} f$ as a subset of the edges of $G$. For every objects $v, w, e_{0}$ such that $e_{0}$ joins $v$ to $w$ in $G$ there exists an object $e$ such that $e$ joins $v$ to $w$ in $G$ and $e \in E_{1}$ and for every object $e^{\prime}$ such that $e^{\prime}$ joins $v$ to $w$ in $G$ and $e^{\prime} \in E_{1}$ holds $e^{\prime}=e$.

(74) Let us consider a non-multi graph $G$, and a representative selection of the parallel edges $E$ of $G$. Then $E=$ the edges of $G$.

(75) Let us consider a graph $G$. Suppose there exists a representative selection of the parallel edges $E$ of $G$ such that $E=$ the edges of $G$. Then $G$ is nonmulti.

(76) Let us consider a non-directed-multi graph $G$, and a representative selection of the directed-parallel edges $E$ of $G$. Then $E=$ the edges of G.

(77) Let us consider a graph $G$. Suppose there exists a representative selection of the directed-parallel edges $E$ of $G$ such that $E=$ the edges of $G$. Then $G$ is non-directed-multi.

(78) Let us consider a graph $G_{1}$, a subgraph $G_{2}$ of $G_{1}$, and a representative selection of the parallel edges $E$ of $G_{1}$. Suppose $E \subseteq$ the edges of $G_{2}$. Then $E$ is a representative selection of the parallel edges of $G_{2}$.

(79) Let us consider a graph $G_{1}$, a subgraph $G_{2}$ of $G_{1}$, and a representative selection of the directed-parallel edges $E$ of $G_{1}$. Suppose $E \subseteq$ the edges of $G_{2}$. Then $E$ is a representative selection of the directed-parallel edges of $G_{2}$.

(80) Let us consider a graph $G_{1}$, a subgraph $G_{2}$ of $G_{1}$, and a representative selection of the parallel edges $E_{2}$ of $G_{2}$. Then there exists a representative selection of the parallel edges $E_{1}$ of $G_{1}$ such that $E_{2}=E_{1} \cap$ (the edges of $\left.G_{2}\right)$.

Proof: Set $A=\left\{\left\{e\right.\right.$, where $e$ is an element of the edges of $G_{1}: e$ joins $v_{1}$ 
and $v_{2}$ in $\left.G_{1}\right\}$, where $v_{1}, v_{2}$ are vertices of $G_{1}$ : there exists an object $e_{0}$ such that $e_{0}$ joins $v_{1}$ and $v_{2}$ in $G_{1}$ and for every object $e_{0}$ such that $e_{0}$ joins $v_{1}$ and $v_{2}$ in $G_{1}$ holds $\left.e_{0} \notin E_{2}\right\}$. Define $\mathcal{P}$ [object, object] $\equiv$ there exists a non empty set $S$ such that $\$_{1}=S$ and $\$_{2}=$ the element of $S$. For every object $x$ such that $x \in A$ there exists an object $y$ such that $\mathcal{P}[x, y]$. Consider $f$ being a function such that $\operatorname{dom} f=A$ and for every object $x$ such that $x \in A$ holds $\mathcal{P}[x, f(x)]$. For every object $e$ such that $e \in \operatorname{rng} f$ holds $e \in$ the edges of $G_{1}$. Reconsider $E_{1}=E_{2} \cup \operatorname{rng} f$ as a subset of the edges of $G_{1}$. For every objects $v, w, e_{0}$ such that $e_{0}$ joins $v$ and $w$ in $G_{1}$ there exists an object $e$ such that $e$ joins $v$ and $w$ in $G_{1}$ and $e \in E_{1}$ and for every object $e^{\prime}$ such that $e^{\prime}$ joins $v$ and $w$ in $G_{1}$ and $e^{\prime} \in E_{1}$ holds $e^{\prime}=e$. For every object $x, x \in E_{2}$ iff $x \in E_{1}$ and $x \in$ the edges of $G_{2}$.

(81) Let us consider a graph $G_{1}$, a subgraph $G_{2}$ of $G_{1}$, and a representative selection of the directed-parallel edges $E_{2}$ of $G_{2}$. Then there exists a representative selection of the directed-parallel edges $E_{1}$ of $G_{1}$ such that $E_{2}=E_{1} \cap\left(\right.$ the edges of $\left.G_{2}\right)$.

Proof: Set $A=\left\{\left\{e\right.\right.$, where $e$ is an element of the edges of $G_{1}: e$ joins $v_{1}$ to $v_{2}$ in $\left.G_{1}\right\}$, where $v_{1}, v_{2}$ are vertices of $G_{1}$ : there exists an object $e_{0}$ such that $e_{0}$ joins $v_{1}$ to $v_{2}$ in $G_{1}$ and for every object $e_{0}$ such that $e_{0}$ joins $v_{1}$ to $v_{2}$ in $G_{1}$ holds $\left.e_{0} \notin E_{2}\right\}$. Define $\mathcal{P}$ [object, object] $\equiv$ there exists a non empty set $S$ such that $\$_{1}=S$ and $\$_{2}=$ the element of $S$. For every object $x$ such that $x \in A$ there exists an object $y$ such that $\mathcal{P}[x, y]$. Consider $f$ being a function such that $\operatorname{dom} f=A$ and for every object $x$ such that $x \in A$ holds $\mathcal{P}[x, f(x)]$. For every object $e$ such that $e \in \operatorname{rng} f$ holds $e \in$ the edges of $G_{1}$. Reconsider $E_{1}=E_{2} \cup \operatorname{rng} f$ as a subset of the edges of $G_{1}$. For every objects $v, w, e_{0}$ such that $e_{0}$ joins $v$ to $w$ in $G_{1}$ there exists an object $e$ such that $e$ joins $v$ to $w$ in $G_{1}$ and $e \in E_{1}$ and for every object $e^{\prime}$ such that $e^{\prime}$ joins $v$ to $w$ in $G_{1}$ and $e^{\prime} \in E_{1}$ holds $e^{\prime}=e$. For every object $x, x \in E_{2}$ iff $x \in E_{1}$ and $x \in$ the edges of $G_{2}$.

(82) Let us consider a graph $G_{1}$, a representative selection of the parallel edges $E_{1}$ of $G_{1}$, a subgraph $G_{2}$ of $G_{1}$ induced by the vertices of $G_{1}$ and $E_{1}$, and a representative selection of the parallel edges $E_{2}$ of $G_{2}$. Then $E_{1}=E_{2}$.

Proof: For every object $e$ such that $e \in E_{1}$ holds $e \in E_{2}$.

(83) Let us consider a graph $G_{1}$, a representative selection of the directedparallel edges $E_{1}$ of $G_{1}$, a subgraph $G_{2}$ of $G_{1}$ induced by the vertices of $G_{1}$ and $E_{1}$, and a representative selection of the directed-parallel edges $E_{2}$ of $G_{2}$. Then $E_{1}=E_{2}$.

Proof: For every object $e$ such that $e \in E_{1}$ holds $e \in E_{2}$. 
(84) Let us consider a graph $G_{1}$, a representative selection of the directedparallel edges $E_{1}$ of $G_{1}$, a subgraph $G_{2}$ of $G_{1}$ induced by the vertices of $G_{1}$ and $E_{1}$, and a representative selection of the parallel edges $E_{2}$ of $G_{2}$. Then

(i) $E_{2} \subseteq E_{1}$, and

(ii) $E_{2}$ is a representative selection of the parallel edges of $G_{1}$.

Let us consider a graph $G$ and representative selections of the parallel edges $E_{1}, E_{2}$ of $G$. Now we state the propositions:

(85) There exists a one-to-one function $f$ such that

(i) $\operatorname{dom} f=E_{1}$, and

(ii) $\operatorname{rng} f=E_{2}$, and

(iii) for every objects $e, v, w$ such that $e \in E_{1}$ holds $e$ joins $v$ and $w$ in $G$ iff $f(e)$ joins $v$ and $w$ in $G$.

Proof: Define $\mathcal{P}$ [object, object $] \equiv \$_{2} \in E_{2}$ and there exist objects $v, w$ such that $\$_{1}$ joins $v$ and $w$ in $G$ and $\$_{2}$ joins $v$ and $w$ in $G$. For every objects $x, y_{1}, y_{2}$ such that $x \in E_{1}$ and $\mathcal{P}\left[x, y_{1}\right]$ and $\mathcal{P}\left[x, y_{2}\right]$ holds $y_{1}=y_{2}$. For every object $x$ such that $x \in E_{1}$ there exists an object $y$ such that $\mathcal{P}[x, y]$. Consider $f$ being a function such that $\operatorname{dom} f=E_{1}$ and for every object $x$ such that $x \in E_{1}$ holds $\mathcal{P}[x, f(x)]$. Consider $v_{0}, w_{0}$ being objects such that $e$ joins $v_{0}$ and $w_{0}$ in $G$ and $f(e)$ joins $v_{0}$ and $w_{0}$ in $G$.

(86) $\overline{\overline{E_{1}}}=\overline{\overline{E_{2}}}$. The theorem is a consequence of (85).

Let us consider a graph $G$ and representative selections of the directedparallel edges $E_{1}, E_{2}$ of $G$. Now we state the propositions:

(87) There exists a one-to-one function $f$ such that

(i) $\operatorname{dom} f=E_{1}$, and

(ii) $\operatorname{rng} f=E_{2}$, and

(iii) for every objects $e, v, w$ such that $e \in E_{1}$ holds $e$ joins $v$ to $w$ in $G$ iff $f(e)$ joins $v$ to $w$ in $G$.

Proof: Define $\mathcal{P}$ [object, object] $\equiv \$_{2} \in E_{2}$ and there exist objects $v, w$ such that $\$_{1}$ joins $v$ to $w$ in $G$ and $\$_{2}$ joins $v$ to $w$ in $G$. For every objects $x, y_{1}, y_{2}$ such that $x \in E_{1}$ and $\mathcal{P}\left[x, y_{1}\right]$ and $\mathcal{P}\left[x, y_{2}\right]$ holds $y_{1}=y_{2}$. For every object $x$ such that $x \in E_{1}$ there exists an object $y$ such that $\mathcal{P}[x, y]$. Consider $f$ being a function such that $\operatorname{dom} f=E_{1}$ and for every object $x$ such that $x \in E_{1}$ holds $\mathcal{P}[x, f(x)]$. Consider $v_{0}, w_{0}$ being objects such that $e$ joins $v_{0}$ to $w_{0}$ in $G$ and $f(e)$ joins $v_{0}$ to $w_{0}$ in $G . v_{0}=v$ and $w_{0}=w$.

(88) $\overline{\overline{E_{1}}}=\overline{\overline{E_{2}}}$. The theorem is a consequence of (87). 
Let $G$ be a graph.

A subgraph of $G$ with parallel edges removed is a subgraph of $G$ defined by

(Def. 7) there exists a representative selection of the parallel edges $E$ of $G$ such that $i t$ is a subgraph of $G$ induced by the vertices of $G$ and $E$.

A subgraph of $G$ with directed-parallel edges removed is a subgraph of $G$ defined by

(Def. 8) there exists a representative selection of the directed-parallel edges $E$ of $G$ such that it is a subgraph of $G$ induced by the vertices of $G$ and $E$.

Observe that every subgraph of $G$ with parallel edges removed is spanning and non-multi and every subgraph of $G$ with directed-parallel edges removed is spanning and non-directed-multi and there exists a subgraph of $G$ with parallel edges removed which is plain and there exists a subgraph of $G$ with directedparallel edges removed which is plain.

Let $G$ be a loopless graph. Let us observe that every subgraph of $G$ with parallel edges removed is simple and every subgraph of $G$ with directed-parallel edges removed is directed-simple.

Now we state the propositions:

(89) Let us consider a non-multi graph $G_{1}$, and a graph $G_{2}$. Then $G_{1} \approx G_{2}$ if and only if $G_{2}$ is a subgraph of $G_{1}$ with parallel edges removed. The theorem is a consequence of (74).

(90) Let us consider a non-directed-multi graph $G_{1}$, and a graph $G_{2}$. Then $G_{1} \approx G_{2}$ if and only if $G_{2}$ is a subgraph of $G_{1}$ with directed-parallel edges removed. The theorem is a consequence of (76).

(91) Let us consider graphs $G_{1}, G_{2}$, and a subgraph $G_{3}$ of $G_{1}$ with parallel edges removed. If $G_{1} \approx G_{2}$, then $G_{3}$ is a subgraph of $G_{2}$ with parallel edges removed. The theorem is a consequence of (78).

(92) Let us consider graphs $G_{1}, G_{2}$, and a subgraph $G_{3}$ of $G_{1}$ with directedparallel edges removed. Suppose $G_{1} \approx G_{2}$. Then $G_{3}$ is a subgraph of $G_{2}$ with directed-parallel edges removed. The theorem is a consequence of (79).

(93) Let us consider graphs $G_{1}, G_{2}$, and a subgraph $G_{3}$ of $G_{1}$ with parallel edges removed. If $G_{2} \approx G_{3}$, then $G_{2}$ is a subgraph of $G_{1}$ with parallel edges removed.

(94) Let us consider graphs $G_{1}, G_{2}$, and a subgraph $G_{3}$ of $G_{1}$ with directedparallel edges removed. Suppose $G_{2} \approx G_{3}$. Then $G_{2}$ is a subgraph of $G_{1}$ with directed-parallel edges removed.

Let us consider a graph $G_{1}$ and a subgraph $G_{2}$ of $G_{1}$ with directed-parallel edges removed. Now we state the propositions: 
(95) Every subgraph of $G_{2}$ with parallel edges removed is a subgraph of $G_{1}$ with parallel edges removed. The theorem is a consequence of (84).

(96) There exists a subgraph $G_{3}$ of $G_{1}$ with parallel edges removed such that $G_{3}$ is a subgraph of $G_{2}$ with parallel edges removed. The theorem is a consequence of (72) and (78).

(97) Let us consider a graph $G_{1}$, and a subgraph $G_{3}$ of $G_{1}$ with parallel edges removed. Then there exists a subgraph $G_{2}$ of $G_{1}$ with directedparallel edges removed such that $G_{3}$ is a subgraph of $G_{2}$ with parallel edges removed. The theorem is a consequence of (73) and (78).

Let $G$ be a complete graph. Let us observe that every subgraph of $G$ with parallel edges removed is complete and every subgraph of $G$ with directedparallel edges removed is complete.

Now we state the propositions:

(98) Let us consider a graph $G_{1}$, a subgraph $G_{2}$ of $G_{1}$ with parallel edges removed, and a walk $W_{1}$ of $G_{1}$. Then there exists a walk $W_{2}$ of $G_{2}$ such that $W_{1}$.vertexSeq ()$=W_{2}$.vertexSeq () .

Proof: Define $\mathcal{P}$ [walk of $\left.G_{1}\right] \equiv$ there exists a walk $W_{2}$ of $G_{2}$ such that $\$_{1}$.vertexSeq ()$=W_{2}$.vertexSeq () . For every trivial walk $W$ of $G_{1}, \mathcal{P}[W]$. For every walk $W$ of $G_{1}$ and for every object $e$ such that

$e \in W$.last().edgesInOut() and $\mathcal{P}[W]$ holds $\mathcal{P}[W$.addEdge $(e)]$. For every walk $W_{1}$ of $G_{1}, \mathcal{P}\left[W_{1}\right]$.

(99) Let us consider a graph $G_{1}$, a subgraph $G_{2}$ of $G_{1}$ with directed-parallel edges removed, and a walk $W_{1}$ of $G_{1}$. Then there exists a walk $W_{2}$ of $G_{2}$ such that $W_{1}$.vertexSeq ()$=W_{2}$.vertexSeq () . The theorem is a consequence of (95) and (98).

(100) Let us consider a graph $G_{1}$, a subgraph $G_{2}$ of $G_{1}$ with parallel edges removed, a vertex $v_{1}$ of $G_{1}$, and a vertex $v_{2}$ of $G_{2}$. If $v_{1}=v_{2}$, then $G_{1}$.reachableFrom $\left(v_{1}\right)=G_{2}$.reachableFrom $\left(v_{2}\right)$. The theorem is a consequence of (35).

(101) Let us consider a graph $G_{1}$, a subgraph $G_{2}$ of $G_{1}$ with directed-parallel edges removed, a vertex $v_{1}$ of $G_{1}$, and a vertex $v_{2}$ of $G_{2}$. If $v_{1}=v_{2}$, then $G_{1}$.reachableFrom $\left(v_{1}\right)=G_{2}$.reachableFrom $\left(v_{2}\right)$. The theorem is a consequence of (35).

Let $G$ be a connected graph. Note that every subgraph of $G$ with parallel edges removed is connected and every subgraph of $G$ with directed-parallel edges removed is connected.

Let $G$ be a non connected graph. One can verify that every subgraph of $G$ with parallel edges removed is non connected and every subgraph of $G$ with directed-parallel edges removed is non connected. 
Now we state the propositions:

(102) Let us consider a graph $G_{1}$, and a subgraph $G_{2}$ of $G_{1}$ with parallel edges removed. Then $G_{1}$.componentSet ()$=G_{2}$. componentSet(). The theorem is a consequence of (100) and (37).

(103) Let us consider a graph $G_{1}$, and a subgraph $G_{2}$ of $G_{1}$ with directedparallel edges removed. Then $G_{1}$.componentSet ()$=G_{2} \cdot \operatorname{componentSet}()$. The theorem is a consequence of (101) and (37).

(104) Let us consider a graph $G_{1}$, and a subgraph $G_{2}$ of $G_{1}$ with parallel edges removed. Then $G_{1}$.numComponents ()$=G_{2}$.numComponents(). The theorem is a consequence of (100) and (38).

(105) Let us consider a graph $G_{1}$, and a subgraph $G_{2}$ of $G_{1}$ with directedparallel edges removed. Then $G_{1}$.numComponents ()$=$ $G_{2}$.numComponents(). The theorem is a consequence of (101) and (38).

(106) Let us consider a graph $G_{1}$, and a subgraph $G_{2}$ of $G_{1}$ with parallel edges removed. Then $G_{1}$ is chordal if and only if $G_{2}$ is chordal. The theorem is a consequence of (98), (30), (32), and (29).

(107) Let us consider a graph $G_{1}$, and a subgraph $G_{2}$ of $G_{1}$ with directedparallel edges removed. Then $G_{1}$ is chordal if and only if $G_{2}$ is chordal. The theorem is a consequence of (95) and (106).

Let $G$ be a chordal graph. Note that every subgraph of $G$ with parallel edges removed is chordal and every subgraph of $G$ with directed-parallel edges removed is chordal.

Now we state the propositions:

(108) Let us consider a graph $G_{1}$, a set $v$, a subgraph $G_{2}$ of $G_{1}$ with parallel edges removed, and a subgraph $G_{3}$ of $G_{1}$ with vertex $v$ removed. Then every subgraph of $G_{2}$ with vertex $v$ removed is a subgraph of $G_{3}$ with parallel edges removed. The theorem is a consequence of (93) and (91).

(109) Let us consider a graph $G_{1}$, a subgraph $G_{2}$ of $G_{1}$ with parallel edges removed, a vertex $v_{1}$ of $G_{1}$, and a vertex $v_{2}$ of $G_{2}$. If $v_{1}=v_{2}$, then $v_{1}$ is cut-vertex iff $v_{2}$ is cut-vertex. The theorem is a consequence of (108) and (104).

(110) Let us consider a graph $G_{1}$, a subgraph $G_{2}$ of $G_{1}$ with directed-parallel edges removed, a vertex $v_{1}$ of $G_{1}$, and a vertex $v_{2}$ of $G_{2}$. If $v_{1}=v_{2}$, then $v_{1}$ is cut-vertex iff $v_{2}$ is cut-vertex. The theorem is a consequence of (95) and (109).

(111) Let us consider a graph $G_{1}$, a subgraph $G_{2}$ of $G_{1}$ with parallel edges removed, a vertex $v_{1}$ of $G_{1}$, and a vertex $v_{2}$ of $G_{2}$. If $v_{1}=v_{2}$, then $v_{1}$ is isolated iff $v_{2}$ is isolated. 
Proof: $v_{1}$.edgesInOut ()$=\emptyset$.

(112) Let us consider a graph $G_{1}$, a subgraph $G_{2}$ of $G_{1}$ with directed-parallel edges removed, a vertex $v_{1}$ of $G_{1}$, and a vertex $v_{2}$ of $G_{2}$. If $v_{1}=v_{2}$, then $v_{1}$ is isolated iff $v_{2}$ is isolated. The theorem is a consequence of (95) and (111).

(113) Let us consider a graph $G_{1}$, a subgraph $G_{2}$ of $G_{1}$ with parallel edges removed, a vertex $v_{1}$ of $G_{1}$, and a vertex $v_{2}$ of $G_{2}$. If $v_{1}=v_{2}$ and $v_{1}$ is endvertex, then $v_{2}$ is endvertex. The theorem is a consequence of (111).

(114) Let us consider a graph $G_{1}$, a subgraph $G_{2}$ of $G_{1}$ with directed-parallel edges removed, a vertex $v_{1}$ of $G_{1}$, and a vertex $v_{2}$ of $G_{2}$. If $v_{1}=v_{2}$ and $v_{1}$ is endvertex, then $v_{2}$ is endvertex. The theorem is a consequence of (112).

Let $G$ be a graph. A simple graph of $G$ is a subgraph of $G$ defined by

(Def. 9) there exists a representative selection of the parallel edges $E$ of $G$ such that $i t$ is a subgraph of $G$ induced by the vertices of $G$ and $E \backslash(G \cdot \operatorname{loops}())$.

A directed-simple graph of $G$ is a subgraph of $G$ defined by

(Def. 10) there exists a representative selection of the directed-parallel edges $E$ of $G$ such that it is a subgraph of $G$ induced by the vertices of $G$ and $E \backslash(G \cdot \operatorname{loops}())$.

Now we state the propositions:

(115) Let us consider a graph $G_{1}$, and a subgraph $G_{2}$ of $G_{1}$ with parallel edges removed. Then every subgraph of $G_{2}$ with loops removed is a simple graph of $G_{1}$. The theorem is a consequence of (48).

(116) Let us consider a graph $G_{1}$, and a subgraph $G_{2}$ of $G_{1}$ with directedparallel edges removed. Then every subgraph of $G_{2}$ with loops removed is a directed-simple graph of $G_{1}$. The theorem is a consequence of (48).

Let us consider a graph $G_{1}$ and a subgraph $G_{2}$ of $G_{1}$ with loops removed. Now we state the propositions:

(117) Every subgraph of $G_{2}$ with parallel edges removed is a simple graph of $G_{1}$. The theorem is a consequence of (80).

(118) Every subgraph of $G_{2}$ with directed-parallel edges removed is a directedsimple graph of $G_{1}$. The theorem is a consequence of (81).

(119) Let us consider a graph $G_{1}$, and a simple graph $G_{3}$ of $G_{1}$. Then there exists a subgraph $G_{2}$ of $G_{1}$ with parallel edges removed such that $G_{3}$ is a subgraph of $G_{2}$ with loops removed.

Proof: Consider $E$ being a representative selection of the parallel edges of $G_{1}$ such that $G_{3}$ is a subgraph of $G_{1}$ induced by the vertices of $G_{1}$ and $E \backslash\left(G_{1}\right.$.loops ()$)$. Set $G_{2}=$ the subgraph of $G_{1}$ induced by the vertices of 
$G_{1}$ and $E$. For every object $e, e \in$ the edges of $G_{3}$ iff $e \in$ (the edges of $\left.G_{2}\right) \backslash\left(G_{2} \cdot \operatorname{loops}()\right)$.

(120) Let us consider a graph $G_{1}$, and a directed-simple graph $G_{3}$ of $G_{1}$. Then there exists a subgraph $G_{2}$ of $G_{1}$ with directed-parallel edges removed such that $G_{3}$ is a subgraph of $G_{2}$ with loops removed.

Proof: Consider $E$ being a representative selection of the directed-parallel edges of $G_{1}$ such that $G_{3}$ is a subgraph of $G_{1}$ induced by the vertices of $G_{1}$ and $E \backslash\left(G_{1}\right.$.loops ()$)$. Set $G_{2}=$ the subgraph of $G_{1}$ induced by the vertices of $G_{1}$ and $E$. For every object $e, e \in$ the edges of $G_{3}$ iff $e \in$ (the edges of $\left.G_{2}\right) \backslash\left(G_{2} \cdot \operatorname{loops}()\right)$.

Let us consider a graph $G_{1}$ and a subgraph $G_{2}$ of $G_{1}$ with loops removed. Now we state the propositions:

(121) Every simple graph of $G_{1}$ is a subgraph of $G_{2}$ with parallel edges removed.

(122) Every directed-simple graph of $G_{1}$ is a subgraph of $G_{2}$ with directedparallel edges removed. The theorem is a consequence of (45) and (6).

Let us consider a loopless graph $G_{1}$ and a graph $G_{2}$. Now we state the propositions:

(123) $\quad G_{2}$ is a simple graph of $G_{1}$ if and only if $G_{2}$ is a subgraph of $G_{1}$ with parallel edges removed.

(124) $G_{2}$ is a directed-simple graph of $G_{1}$ if and only if $G_{2}$ is a subgraph of $G_{1}$ with directed-parallel edges removed.

(125) Let us consider a non-multi graph $G_{1}$, and a graph $G_{2}$. Then $G_{2}$ is a simple graph of $G_{1}$ if and only if $G_{2}$ is a subgraph of $G_{1}$ with loops removed. The theorem is a consequence of (74).

(126) Let us consider a non-directed-multi graph $G_{1}$, and a graph $G_{2}$. Then $G_{2}$ is a directed-simple graph of $G_{1}$ if and only if $G_{2}$ is a subgraph of $G_{1}$ with loops removed. The theorem is a consequence of (76).

Let $G$ be a graph. Note that every simple graph of $G$ is spanning, loopless, non-multi, and simple and every directed-simple graph of $G$ is spanning, loopless, non-directed-multi, and directed-simple and there exists a simple graph of $G$ which is plain and there exists a directed-simple graph of $G$ which is plain.

Now we state the propositions:

(127) Let us consider a simple graph $G_{1}$, and a graph $G_{2}$. Then $G_{1} \approx G_{2}$ if and only if $G_{2}$ is a simple graph of $G_{1}$. The theorem is a consequence of (74).

(128) Let us consider a directed-simple graph $G_{1}$, and a graph $G_{2}$. Then $G_{1} \approx$ $G_{2}$ if and only if $G_{2}$ is a directed-simple graph of $G_{1}$. The theorem is 
a consequence of $(76)$.

(129) Let us consider graphs $G_{1}, G_{2}$, and a simple graph $G_{3}$ of $G_{1}$. If $G_{1} \approx G_{2}$, then $G_{3}$ is a simple graph of $G_{2}$. The theorem is a consequence of (50) and (78).

(130) Let us consider graphs $G_{1}, G_{2}$, and a directed-simple graph $G_{3}$ of $G_{1}$. If $G_{1} \approx G_{2}$, then $G_{3}$ is a directed-simple graph of $G_{2}$. The theorem is a consequence of (50) and (79).

(131) Let us consider graphs $G_{1}, G_{2}$, and a simple graph $G_{3}$ of $G_{1}$. If $G_{2} \approx G_{3}$, then $G_{2}$ is a simple graph of $G_{1}$.

(132) Let us consider graphs $G_{1}, G_{2}$, and a directed-simple graph $G_{3}$ of $G_{1}$. If $G_{2} \approx G_{3}$, then $G_{2}$ is a directed-simple graph of $G_{1}$.

Let us consider a graph $G_{1}$ and a directed-simple graph $G_{2}$ of $G_{1}$. Now we state the propositions:

(133) Every simple graph of $G_{2}$ is a simple graph of $G_{1}$. The theorem is a consequence of (122), (123), (95), and (117).

(134) There exists a simple graph $G_{3}$ of $G_{1}$ such that $G_{3}$ is a simple graph of $G_{2}$. The theorem is a consequence of (122), (96), (117), and (123).

(135) Let us consider a graph $G_{1}$, and a simple graph $G_{3}$ of $G_{1}$. Then there exists a directed-simple graph $G_{2}$ of $G_{1}$ such that $G_{3}$ is a simple graph of $G_{2}$. The theorem is a consequence of (121), (97), (118), and (123).

Let $G$ be a complete graph. Observe that every simple graph of $G$ is complete and every directed-simple graph of $G$ is complete.

Now we state the propositions:

(136) Let us consider a graph $G_{1}$, a simple graph $G_{2}$ of $G_{1}$, and a walk $W_{1}$ of $G_{1}$. Then there exists a walk $W_{2}$ of $G_{2}$ such that $W_{2}$ is walk from $W_{1}$.first() to $W_{1} \cdot \operatorname{last}()$. The theorem is a consequence of (119) and (61).

(137) Let us consider a graph $G_{1}$, a directed-simple graph $G_{2}$ of $G_{1}$, and a walk $W_{1}$ of $G_{1}$. Then there exists a walk $W_{2}$ of $G_{2}$ such that $W_{2}$ is walk from $W_{1}$.first() to $W_{1}$.last(). The theorem is a consequence of (133) and (136).

(138) Let us consider a graph $G_{1}$, a simple graph $G_{2}$ of $G_{1}$, a vertex $v_{1}$ of $G_{1}$, and a vertex $v_{2}$ of $G_{2}$. If $v_{1}=v_{2}$, then $G_{1}$.reachableFrom $\left(v_{1}\right)=$ $G_{2}$.reachableFrom $\left(v_{2}\right)$. The theorem is a consequence of (136) and (35).

(139) Let us consider a graph $G_{1}$, a directed-simple graph $G_{2}$ of $G_{1}$, a vertex $v_{1}$ of $G_{1}$, and a vertex $v_{2}$ of $G_{2}$. If $v_{1}=v_{2}$, then $G_{1}$.reachableFrom $\left(v_{1}\right)=$ $G_{2}$.reachableFrom $\left(v_{2}\right)$. The theorem is a consequence of (137) and (35).

Let $G$ be a connected graph. Observe that every simple graph of $G$ is connected and every directed-simple graph of $G$ is connected. 
Let $G$ be a non connected graph. One can verify that every simple graph of $G$ is non connected and every directed-simple graph of $G$ is non connected.

Now we state the propositions:

(140) Let us consider a graph $G_{1}$, and a simple graph $G_{2}$ of $G_{1}$.

Then $G_{1}$.componentSet ()$=G_{2}$.componentSet () . The theorem is a consequence of (138) and (37).

(141) Let us consider a graph $G_{1}$, and a directed-simple graph $G_{2}$ of $G_{1}$. Then $G_{1}$.componentSet ()$=G_{2}$.componentSet(). The theorem is a consequence of (139) and (37).

(142) Let us consider a graph $G_{1}$, and a simple graph $G_{2}$ of $G_{1}$. Then $G_{1}$.numComponents ()$=G_{2}$.numComponents () . The theorem is a consequence of (138) and (38).

(143) Let us consider a graph $G_{1}$, and a directed-simple graph $G_{2}$ of $G_{1}$. Then $G_{1}$.numComponents ()$=G_{2}$.numComponents () . The theorem is a consequence of (139) and (38).

(144) Let us consider a graph $G_{1}$, and a simple graph $G_{2}$ of $G_{1}$. Then $G_{1}$ is chordal if and only if $G_{2}$ is chordal. The theorem is a consequence of (119), (65), and (106).

(145) Let us consider a graph $G_{1}$, and a directed-simple graph $G_{2}$ of $G_{1}$. Then $G_{1}$ is chordal if and only if $G_{2}$ is chordal. The theorem is a consequence of (120), (65), and (107).

Let $G$ be a chordal graph. One can check that every simple graph of $G$ is chordal and every directed-simple graph of $G$ is chordal.

Now we state the propositions:

(146) Let us consider a graph $G_{1}$, a simple graph $G_{2}$ of $G_{1}$, a vertex $v_{1}$ of $G_{1}$, and a vertex $v_{2}$ of $G_{2}$. If $v_{1}=v_{2}$, then $v_{1}$ is cut-vertex iff $v_{2}$ is cut-vertex. The theorem is a consequence of (119), (67), and (109).

(147) Let us consider a graph $G_{1}$, a directed-simple graph $G_{2}$ of $G_{1}$, a vertex $v_{1}$ of $G_{1}$, and a vertex $v_{2}$ of $G_{2}$. If $v_{1}=v_{2}$, then $v_{1}$ is cut-vertex iff $v_{2}$ is cut-vertex. The theorem is a consequence of (120), (67), and (110).

(148) Let us consider a loopless graph $G_{1}$, a simple graph $G_{2}$ of $G_{1}$, a vertex $v_{1}$ of $G_{1}$, and a vertex $v_{2}$ of $G_{2}$. If $v_{1}=v_{2}$, then $v_{1}$ is isolated iff $v_{2}$ is isolated. The theorem is a consequence of (119), (58), and (111).

(149) Let us consider a loopless graph $G_{1}$, a directed-simple graph $G_{2}$ of $G_{1}$, a vertex $v_{1}$ of $G_{1}$, and a vertex $v_{2}$ of $G_{2}$. If $v_{1}=v_{2}$, then $v_{1}$ is isolated iff $v_{2}$ is isolated. The theorem is a consequence of (120), (58), and (112).

(150) Let us consider a graph $G_{1}$, a simple graph $G_{2}$ of $G_{1}$, a vertex $v_{1}$ of $G_{1}$, and a vertex $v_{2}$ of $G_{2}$. If $v_{1}=v_{2}$ and $v_{1}$ is endvertex, then $v_{2}$ is endvertex. 
The theorem is a consequence of (119), (113), and (68).

(151) Let us consider a graph $G_{1}$, a directed-simple graph $G_{2}$ of $G_{1}$, a vertex $v_{1}$ of $G_{1}$, and a vertex $v_{2}$ of $G_{2}$. If $v_{1}=v_{2}$ and $v_{1}$ is endvertex, then $v_{2}$ is endvertex. The theorem is a consequence of (120), (114), and (68).

\section{REFERENCES}

[1] Grzegorz Bancerek. Introduction to trees Formalized Mathematics, 1(2):421-427, 1990.

[2] Grzegorz Bancerek, Czesław Byliński, Adam Grabowski, Artur Korniłowicz, Roman Matuszewski, Adam Naumowicz, and Karol Pąk. The role of the Mizar Mathematical Library for interactive proof development in Mizar. Journal of Automated Reasoning, 61(1):9-32, 2018. do1:10.1007/s10817-017-9440-6

[3] Lowell W. Beineke and Robin J. Wilson, editors. Selected Topics in Graph Theory. Academic Press, London, 1978. ISBN 0-12-086250-6.

[4] John Adrian Bondy and U. S. R. Murty. Graph Theory. Graduate Texts in Mathematics, 244. Springer, New York, 2008. ISBN 978-1-84628-969-9.

[5] Adam Grabowski, Artur Korniłowicz, and Adam Naumowicz. Four decades of Mizar. Journal of Automated Reasoning, 55(3):191-198, 2015. doi 10.1007/s10817-015-9345-1

[6] Krzysztof Hryniewiecki. Graphs. Formalized Mathematics, 2(3):365-370, 1991.

[7] Gilbert Lee. Weighted and labeled graphs Formalized Mathematics, 13(2):279-293, 2005.

[8] Gilbert Lee and Piotr Rudnicki. Alternative graph structures Formalized Mathematics, 13(2):235-252, 2005.

[9] Piotr Rudnicki and Lorna Stewart. Simple graphs as simplicial complexes: the Mycielskian of a graph. Formalized Mathematics, 20(2):161-174, 2012. doi 10.2478/v10037-012-00198.

[10] Yozo Toda. The formalization of simple graphs Formalized Mathematics, 5(1):137-144, 1996.

[11] Klaus Wagner. Graphentheorie. B.I-Hochschultaschenbücher; 248. Bibliograph. Inst., Mannheim, 1970. ISBN 3-411-00248-4.

[12] Robin James Wilson. Introduction to Graph Theory. Oliver \& Boyd, Edinburgh, 1972. ISBN 0-05-002534-1. 